\title{
DPP-4-Hemmer \\ Kritik an der frühen Nutzenbewertung
}

Es ist weltweit zugelassen und in 25 Ländern verfügbar, aber in Deutschland wird Linagliptin bis auf Weiteres nicht erhältlich sein. Die Preisverhandlungen mit dem GKV-Spitzenverband sind am 26. April gescheitert. Kritik am derzeitigen Vorgehen bei der frühen Nutzenbewertung gab es beim Diabeteskongress in Stuttgart seitens der Deutschen Diabetes-Gesellschaft (DDG), des Deutschen Diabetikerbundes (DDB) sowie der Hersteller des DPP4-Hemmers.

\section{Matthaei: Kein Zweifel am Zusatznutzen von Linagliptin}

DDG-Präsident Prof. Stephan Matthaei widersprach dem Ergebnis des IQWiG, wonach es keinen Beleg für einen Zusatznutzen von Linagliptin (Trajenta ${ }^{\circledR}$ ) gegenüber Sulfonylharnstoffen und Insulin gebe, indem er aus einer Stellungnahme der DDG vom Januar 2012 zitierte: Sowohl wissenschaftlich als auch klinisch gebe es aus Sicht der DDG keinerlei Zweifel am Zusatznutzen der DPP-4-Hemmer bei hypoglykämiegefährdeten und stark übergewichtigen $\mathrm{Pa}$ tienten gegenüber Sulfonylharnstoffen oder Insulin, besonders bei Kombination mit Metformin. Gerade Linagliptin sei bei schwerer Niereninsuffizienz gegenüber Sulfonylharnstoffen vorteilhaft, die dann bekanntlich kontraindiziert sind.

\section{Patientenbelange zu wenig berücksichtigt}

Sabine Westermann vom DDB sieht bei der frühen Nutzenbewertung von Linagliptin Defizite bei der Berücksichtigung der Patientenbelange. Alltagsbedingungen, Bedürfnisse und die Selbstbestimmung der Patienten seien nicht in die Bewertung eingeflossen. Bei der Wahl der zweckmäßigen Vergleichstherapie stünden offenbar die kurzfristigen Kosten für die GKV im Vordergrund. Dabei müssten auch Kosten in anderen Bereichen beachtet werden, etwa wenn es um das Thema Berufsunfähigkeit und Frühberentung von Diabetikern geht.

Dr. Marco Penske vom Unternehmen Boehringer Ingelheim, das den DPP-4-Hemmer in einer weltweiten Allianz mit Lilly vertreibt, nannte Kritikpunkte der Hersteller. Die adäquate Vergleichstherapie sei Sitagliptin, da es in der Therapiehierarchie laut Leitlinien auf derselben Stufe steht wie Linagliptin.

Sulfonylharnstoffe und Insulin waren vom G-BA zunächst unverbindlich als Vergleichswirkstoffe genannt worden, weshalb die Hersteller ein vollständiges Dossier eingereicht hätten, was vom G-BA auch zunächst bestätigt worden sei. Erst die endgültige Wahl von Sulfonylharnstoffen und Insulin als Vergleichstherapie habe dann zur Unvollständigkeit des Dossiers geführt. „Linagliptin wurde der
Zusatznutzen also aus rein formalen Gründen abgesprochen“, so Penske, zumal im Dossier auch Vergleichsdaten zu Sulfonylharnstoffen enthalten seien.

\section{Zugebilligter Tagestherapiepreis sehr niedrig}

Die Diskrepanz zwischen dem zugebilligten Tagestherapiepreis im niedrigen zweistelligen Cent-Bereich und dem für die Hersteller in Deutschland akzeptablen Abgabepreis von 1,20 Euro sei zu groß, sagte Penske, daher habe man von einer Markteinführung Linagliptins abgesehen.

Da es sich bei der frühen Nutzenbewertung um ein lernendes System handele, wünscht er sich im Namen der Hersteller Nachbesserungen: Für die Wahl der Vergleichstherapie sollten auch die Leitlinien der Fachgesellschaften berücksichtigt werden, und die Festlegung sollte vor Beginn des Verfahrens verbindlich sein. Außer harten Endpunkten wie der Mortalität sollten auch patientenrelevante Parameter wie Gewicht und Lebensqualität in die Nutzenbeurteilung eingehen. Zusätzlich müssten die zwischen Hersteller und GKV-Spitzenverband verhandelten Rabatte vertraulich bleiben, um andere Märkte nicht zu beeinflussen. Zu guter Letzt sei die Möglichkeit der schnellen Wiederaufnahme des Beurteilungsverfahrens notwendig, um die Lernkurve nicht auf Kosten der Hersteller zu vollziehen.

Quelle: Pressekonferenz beim Deutschen Diabeteskongress, Stuttgart, 16.5.2012, Veranstalter: Boehringer Ingelheim und Lilly

\section{Kurz notiert}

\section{Internetportal für Diabetiker}

Informationen rund um Typ-1- und Typ-2-Diabetes sind ab sofort unter www.bayerdiabetes.de zu finden. Das neue Online-Portal erklärt zum Beispiel die unterschiedlichen Therapieformen bei Diabetes. Zusätzlich gibt es anschauliche, interaktive und praktische Tipps zur Ernährung und Bewegung. Wer sich über die Blutzuckermesssysteme Contour, Contour USB, Contour Link und Breeze informieren möchte, wird im Produktbereich fündig.

Zahlreiche kostenfreie Ratgeber-Broschüren zum Herunterladen oder Bestellen runden das neue Angebot ab. Bei der kostenfreien TelefonHotline (0800 - 5088822$)$ finden Nutzer montags bis freitags von 8 bis 20 Uhr zudem kompetente Ansprechpartner für ihre Fragen rund um den Diabetes und die Anwendung der Bayer Blutzuckermessgeräten.

Quelle: Bayer Vital 